\title{
The Influence of Restrictive Pulmonary Dysfunction on Osteoporotic Thoracic Vertebral Fractures
}

\author{
Ji-Hun Park, Sang-Min Lee, Seong-Woo Shim, Sung-Nyun Baek, Yong-Soo Choi \\ Department of Orthopedic Surgery, Kwangju Christian Hospital, Gwangju, Korea
}

\begin{abstract}
Study Design: A cross-sectional study.
Purpose: To examine the influence of restrictive pulmonary dysfunction on osteoporotic thoracic vertebral fractures.

Overview of Literature: Osteoporotic thoracic vertebral fractures generally result in an increased kyphotic angle, which in turn may lead to pulmonary function impairment. Impaired pulmonary function could be associated with vertebral fractures. However, an association between osteoporotic thoracic vertebral fractures and pulmonary function remains controversial.

Methods: A total of 96 patients were enrolled in this study, 30 of whom had osteoporotic thoracic vertebral fractures (group 1), 30 with chronic back pain (group 2), and 36 with chronic pulmonary diseases (group 3). Radiologic study of prevalent vertebral fractures, thoracic kyphotic angle, bone mineral density, relaxed expiratory vital capacity, forced vital capacity (FVC), and forced expiratory volume in 1 second (FEV1) in spirometry was investigated.

Results: The mean FVC and FEV1 were $75.66 \% \pm 20.23 \%$ and $79.93 \% \pm 22.48 \%$, respectively, in group $1 ; 84.50 \% \pm 16.25 \%$ and $91.87 \% \pm 21.65 \%$, respectively, in group 2 ; and $91.64 \% \pm 17.53 \%$ and $91.03 \% \pm 23.71 \%$, respectively, in group 3 . Group 1 (patients with osteoporotic thoracic vertebral fracture) had the lowest FVC among the three groups ( $p=0.01)$. Group 1 revealed worse result of pulmonary dysfunction than group 3 (patients with chronic pulmonary diseases) $(p=0.01)$. The average kyphosis angle of the thoracic spine was $26.95^{\circ} \pm 15.17^{\circ}, 36.47^{\circ} \pm 20.08^{\circ}$, and $28.58^{\circ} \pm 10.58^{\circ}$ in groups 1,2 , and 3 , respectively. There was a negative correlation between thoracic kyphosis and FEV1 $(r=-0.309, p=0.01)$.

Conclusions: The results suggest that osteoporotic thoracic vertebral fracture burden could be affected by restrictive pulmonary dysfunction.
\end{abstract}

Keywords: Thoracic spine; Vertebral fracture; Osteoporosis; Respiratory function tests

\section{Introduction}

As the population ages, the incidence of osteoporosisrelated complications continues to increase. Vertebral compression fracture is the most common complication of osteoporosis. The progressive collapse of the fractured osteoporotic vertebral body generally results in an increased kyphotic angle, which in turn may lead to disturbances in pulmonary function. Spinal kyphosis may cause restriction of pulmonary function [1].

Received Feb 21, 2020; Revised May 1, 2020; Accepted May 18, 2020

Corresponding author: Yong-Soo Choi

Department of Orthopedic Surgery, Kwangju Christian Hospital, 37 Yangnim-ro, Nam-gu, Gwangju 61661, Korea

Tel: +82-62-650-5064, Fax: +82-62-650-5066, E-mail: stemcellchoi@gmail.com 
Pulmonary dysfunction may be associated with vertebral fractures. Prevalence of vertebral fractures in patients with chronic obstructive pulmonary disease (COPD) has been reported to be 24\%-79\% [2-8]. Again, the results widely vary depending on demographic data and severity of pulmonary dysfunction of study subjects.

In previous studies investigating the relationship between vertebral fractures and bone mineral density (BMD) in patients with COPD, BMD was not a good predictor of vertebral fracture. Watanabe et al. [9] reported that $79.4 \%$ had prevalent vertebral fracture whereas only $38.8 \%$ had BMD-defined osteoporosis $(\mathrm{T} \leq 2.5)$ in 136 Japanese patients. This result suggested the involvement of BMDindependent mechanisms in COPD patients.

In few studies that analyzed respiratory capacity, an increased thoracic kyphotic angle was found to influence a decline in inspiratory and vital capacity (VC) [10]. Leech et al. [11] reported that modest decline in VC was associated with the degree of kyphotic deformity and the number of thoracic vertebral fractures resulting from spinal osteoporosis [12]. Although kyphotic deformity of the spine is a well-recognized complication of osteoporotic vertebral fractures, there is less interest in its concomitant association with pulmonary dysfunction. In addition, the association between osteoporotic vertebral fractures and pulmonary function remains controversial. This study aimed to examine the influence of restrictive pulmonary dysfunction on osteoporotic thoracic vertebral fractures.

\section{Materials and Methods}

Between January and December 2018, 96 patients who had lateral plain radiograph of the thoracic and lumbar spine, bone densitometry, and spirometry were enrolled in the study. All patients were divided into three groups, i.e., 30 patients with osteoporotic thoracic vertebral fractures (group 1), 30 patients with chronic back pain without osteoporotic vertebral fractures (group 2), and 36 patients with chronic pulmonary diseases (group 3). Vertebral fracture was diagnosed by radiograph and magnetic resonance imaging. In group 1, two patients had fracture at $\mathrm{T} 8$, four at $\mathrm{T} 9$, seven at $\mathrm{T} 10$, nine at T11, and eight at T12. In group 2, chronic back pain was defined as an upper back pain in the thoracic spine area that persists for 12 weeks or longer, even after an underlying cause of acute upper back pain had been treated. In group 3, chronic pulmonary disease was defined as a type of disorder that affects the lungs and other parts of the respiratory system diagnosed by a medical physician; 24 patients had COPD, four had asthma, and eight had other lung conditions. In particular, four patients with asthma were treated with steroids. However, there was no significant difference in mean T-score of BMD between asthma patients (-2.2) and chronic pulmonary disease patients $(-2.1)$. Patients who had acute back pain caused by injury, pathologic fractures, spinal infection, and metastatic cancer were excluded. The average age of patients in group 1 was $73.97 \pm 14.26$ years with five males and 25 females. The average age of patients in group 2 was $73.73 \pm 7.96$ years with five males and 25 females. The average age of patients in group 3 was $72.67 \pm 7.02$ years with six males and 30 females. Body mass index (BMI) was 23.03, 22.79, and $23.21 \mathrm{~kg} / \mathrm{m}^{2}$ in groups 1,2 , and 3, respectively. No significant differences were found in age, sex, or BMI among the three groups $(p>0.05)$ (Table 1$)$. The study was exempted from the requirement to obtain informed consent from the subjects. This study was approved by the Institutional Review Board at the Kwangju Christian Hospital (KCHM-2020-03-002).

$\mathrm{BMD}$, spine radiography, and spirometry were examined. BMD was measured using dual-energy X-ray absorptiometry (Lunar iDXA Fan Beam; GE Healthcare, Chicago, IL, USA) in the lumbar spine because some patients had hip arthroplasty or compression hip screw. The L1-L4 region was measured unless focal artifacts require exclusion of individual vertebra. Measurements of the lumbar spine were analyzed in accordance to the manufacturer's recommendations. All patients underwent whole spine lateral radiograph in a standing position as the standard method, which was performed in an average of 3.1 months after the development of fracture in group 1. At this time, fracture-related pain had no specific influence on the standing posture. To measure the thoracic kyphosis angle, a perpendicular was extended from a line drawn through a superior landmark marking from $\mathrm{T} 4$ and an inferior marking of T12. The mean values of thoracic kyphosis were determined and compared among the three groups. For evaluation of respiratory function, flow volume was measured using an electronic spirometry (Platinum Elite; MGC Diagnostics, Saint Paul, MN, USA). In group 1, fracture-related pain was improved by wearing braces in an average of 3.1 months after the development of fracture. At this time, the brace and fracture-related pain there had no specific influence on the sitting posture. 
Relaxed expiratory VC, forced vital capacity (FVC), forced expiratory volume in 1 second (FEV1), forced expiratory flow (FEF), forced inspiratory vital capacity (FIVC), and FEV1/FVC ratio were used as parameters of pulmonary function. All tests were conducted by the same laboratory technician.

PASW SPSS for Windows software ver. 18.0 (SPSS Inc., Chicago, IL, USA) was used for statistical analyses. A general linear model was used to evaluate the association between osteoporotic vertebral fractures and independent variables such as sex, age, BMI, and BMD. Analysis of variance test was used to determine a significant relationship among the three groups in terms of pulmonary function test and thoracic kyphotic angle. Results were expressed as means \pm standard deviations. Pearson's correlation was used to determine a significant relationship between osteoporotic vertebral fracture and thoracic kyphotic angle, relaxed expiratory VC, FVC, and FEV1. A $p<0.05$ was considered statistically significant.

\section{Results}

Mean age, FVC, and FEV1 were 73.97 years, $75.66 \% \pm 20.23 \%$, and $79.93 \% \pm 22.48 \%$, respectively, in group $1 ; 73.73$ years, $84.50 \% \pm 16.25 \%$, and $91.87 \% \pm 21.65 \%$, respectively, in group 2; and 72.67 years, $91.64 \% \pm 17.53 \%$, and $91.03 \% \pm 23.71 \%$, respectively, in group 3. No significant difference was found in age, sex, and BMI among the three groups ( $p>0.05$ ) (Table 1). Group 1 had the lowest FVC among the three groups $(p=0.01)$. Group 1 revealed worse result of pulmonary dysfunction than group $3(p=0.01)$ (Table 2).

The average kyphosis angle of the thoracic spine was $26.95^{\circ} \pm 15.17^{\circ}, 36.47^{\circ} \pm 20.08^{\circ}$, and $28.58^{\circ} \pm 10.58^{\circ}$ in groups 1, 2, and 3, respectively. Group 2 had severe thoracic kyphosis among the three groups $(p=0.01)$ (Table 3 ). Although group 1 had the lowest kyphotic deformity of the thoracic spine among the three groups, a significant association was found between osteoporotic thoracic vertebral fractures and restrictive pulmonary dysfunction with the lowest FVC $(p=0.01)$. However, there was a negative correlation between thoracic kyphosis and FEV1 in all patients regardless of groups $(r=-0.309, p=0.01$ ) (Fig. $1)$.

In addition, fracture-related pain may affect the results of spirometry. However, FEV1/FVC ratio revealed the best result in group 2 and the worst result in group $3(p=0.02)$.
Table 1. Comparison of basic data among three groups

\begin{tabular}{lcccc} 
Characteristic & $\begin{array}{c}\text { Group 1 } \\
(\mathrm{n}=30)\end{array}$ & $\begin{array}{c}\text { Group 2 } \\
(\mathrm{n}=30)\end{array}$ & $\begin{array}{c}\text { Group 3 } \\
(\mathrm{n}=36)\end{array}$ & $p$-value \\
\hline Gender (male:female) & $1: 5$ & $1: 5$ & $1: 5$ & \\
\hline Age $(\mathrm{yr})$ & $73.97 \pm 14.26$ & $73.73 \pm 7.96$ & $72.67 \pm 7.02$ & 0.562 \\
\hline Body mass index $\left(\mathrm{kg} / \mathrm{m}^{2}\right)$ & $23.03 \pm 3.48$ & $22.79 \pm 3.55$ & $23.21 \pm 3.15$ & 0.683 \\
\hline
\end{tabular}

Values are presented as number or mean \pm standard deviation. Group 1: osteoporotic vertebral fracture; group 2: chronic back pain; and group 3: chronic pulmonary diseases.

Table 2. Comparison of pulmonary function test

\begin{tabular}{lcccc} 
Variable & $\begin{array}{c}\text { Group 1 } \\
(\mathrm{n}=30)\end{array}$ & $\begin{array}{c}\text { Group 2 } \\
(\mathrm{n}=30)\end{array}$ & $\begin{array}{c}\text { Group 3 } \\
(\mathrm{n}=36)\end{array}$ & p-value \\
\hline FVC $(\%)$ & $75.66 \pm 20.23$ & $84.50 \pm 16.25$ & $91.64 \pm 17.53$ & $0.003^{*}$ \\
\hline FEV1 (\%) & $79.93 \pm 22.48$ & $91.87 \pm 21.65$ & $91.03 \pm 23.71$ & 0.081 \\
\hline FEV1/FVC (\%) & $104.62 \pm 12.62$ & $106.67 \pm 14.16$ & $97.69 \pm 15.14$ & $0.028^{*}$ \\
\hline FEF max (\%) & $76.66 \pm 23.58$ & $79.90 \pm 24.67$ & $84.44 \pm 26.17$ & 0.451 \\
\hline FIVC (L) & $2.09 \pm 1.18$ & $2.08 \pm 0.52$ & $2.34 \pm 0.56$ & 0.325 \\
\hline
\end{tabular}

Values are presented as mean \pm standard deviation. Group 1: osteoporotic vertebral fracture; group 2: chronic back pain; group 3: chronic pulmonary diseases. FVC, forced vital capacity; FEV1, forced expiratory volume in 1 second; FEF, forced expiratory flow; FIVC, forced inspiratory vital capacity. " $p<0.05$.

Table 3. Comparison of thoracic kyphotic angle and BMD among three groups

$\begin{array}{ccccc}\text { Variable } & \begin{array}{c}\text { Group } 1 \\ (n=30)\end{array} & \begin{array}{c}\text { Group 2 } \\ (\mathrm{n}=30)\end{array} & \begin{array}{c}\text { Group 3 } \\ (\mathrm{n}=36)\end{array} & p \text {-value }\end{array}$

Thoracic kyphotic angle $\left(^{\circ}\right) \quad 26.95 \pm 15.17 \quad 36.47 \pm 20.08 \quad 28.58 \pm 10.58 \quad 0.001^{*}$

BMD (T-score) $\quad-3.06 \pm 1.01 \quad-2.64 \pm 1.05 \quad-2.13 \pm 0.99 \quad 0.002^{*}$

Group 1: osteoporotic vertebral fracture; group 2: chronic back pain; and group 3: chronic pulmonary diseases. $\mathrm{BMD}$, bone mineral density. " $p<0.05$.

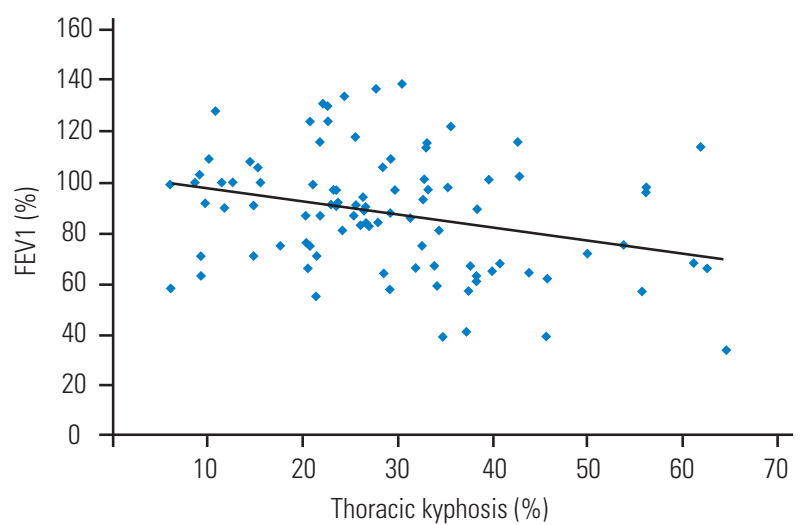

Fig. 1. Correlation of between the thoracic kyphosis and FEV1 $(r=-0.309$, $p=0.01$ ). FEV1, forced expiratory volume in 1 second. 
Group 1 had the lowest BMD $(p=0.01)$ among the three groups. However, no significant correlation was found between BMD and pulmonary function parameters $(p>0.05)$.

\section{Discussion}

Osteoporotic thoracic vertebral fractures could result in restrictive pulmonary dysfunction owing to changes in the thoracic cavity. Thoracic kyphotic deformity may cause restrictive thoracic movement because of mechanical pain and changes in the thoracic cavity, thereby impairing pulmonary function [13]. At the same time, thoracic muscle mass and strength could affect respiratory function [5]. Although kyphotic deformity of the thoracic spine can cause pulmonary dysfunction, the relationship between changes in the thoracic cavity such as kyphotic deformity and pulmonary dysfunction is complex [1].

The influence of thoracic compression fracture burden on reduction in inspiratory time is consistent with reduced lung volume. Krege et al. [4] reported that thoracic spine fracture burden significantly reduced lung volume but not flow, suggesting that thoracic spine fracture burden is associated with restrictive, but not obstructive, lung disease [5]. This study found that osteoporotic vertebral fractures of the thoracic spine were associated with significant decreases in FIVC and inspiratory time. Leech et al. [11] described that FVC declined in thoracic vertebral fractures and reported that thoracic compression fractures with kyphotic deformity resulted in a decline in VC [14]. On the other hand, Yang et al. [1] reported that kyphoplasty improved respiratory function, particularly FVC and maximum voluntary ventilation. Improvement in thoracic kyphosis could also increase thoracic capacity and hence improve restrictive pulmonary dysfunction.

A normal thoracic spine should have a slight kyphotic angle, ranging from $20^{\circ}$ to $45^{\circ}$, particularly around $40^{\circ}$ in elderly people. When the roundness of the upper spine increases past $45^{\circ}$, it is called kyphosis. According to our results, patients with chronic back pain (group 2) had the highest thoracic kyphotic angle $\left(36.47^{\circ}\right.$ of mean thoracic kyphotic angle). In other words, all three groups had relatively normal range of thoracic kyphotic angle even though some cases had hyperkyphosis. However, it was hard to deny that thoracic kyphotic deformity had influence on restrictive pulmonary dysfunction because a negative correlation was found between thoracic kyphosis and FEV1 in all patients regardless of groups $(r=-0.309$, $p=0.01)$. The increase in kyphotic angle of the thoracic spine with age was not unexpected, because of associated changes in BMD and muscular tissues with progression of years. Osteoporotic thoracic vertebral fractures could increase the thoracic kyphotic angle progressively with prevalence of secondary new fractures and its narrowing of the intervertebral discs. In this study, patients with osteoporotic thoracic vertebral fracture (group 1) had the lowest BMD ( -3.06 of mean T-score). However, BMD alone is inadequate in evaluating the risk of osteoporotic fractures [15,16]. Watanabe et al. [5] reported that pulmonary dysfunction was associated with prevalent vertebral fractures and decreased BMD in Japanese postmenopausal women. These results suggest that it is important for the elderly to maintain pulmonary function using pulmonary rehabilitation program, such as health education, breathing techniques, and constant exercise to keep good spinal range of motion and strength of the back-extensor muscle. There are many risk factors for osteoporotic vertebral fractures. Although osteoporotic vertebral fracture in the thoracic spine could result in declined pulmonary function, restrictive pulmonary dysfunction might in turn increase osteoporotic vertebral fracture risk, indicating a complex bidirectional interaction.

This study had some limitations. First, the demographic data were nonhomogeneous, particularly BMD of the study group and the control group. Second, this was a cross-sectional study. Third, the number of patients was too small to evaluate multifactorial risks. Fourth, because lateral plain radiograph of the whole spine and spirometry were taken at an average of 3.1 months after the fracture, there was a possibility of changes in variables in the osteoporotic thoracic vertebral fracture group, such as thoracic kyphotic angle, VC, FVC, FEV1, FEF, and FIVC, resulting from the influence of the fracture itself. However, despite these limitations, this study provided potential areas for future research. To achieve more reliability, a prospective cohort study is needed to investigate the relationship between osteoporotic thoracic vertebral fractures and restrictive pulmonary dysfunction in the general population in the future.

\section{Conclusions}

These results suggest that osteoporotic thoracic vertebral fracture burden could be affected by restrictive pulmonary dysfunction. It is necessary for osteoporotic thoracic 
vertebral fractures in the elderly to maintain pulmonary function using pulmonary rehabilitation program, such as health education, breathing techniques, and constant exercise to keep good spinal range of motion and strength of the back-extensor muscle.

\section{Conflict of Interest}

No potential conflict of interest relevant to this article was reported.

\section{References}

1. Yang HL, Zhao L, Liu J, et al. Changes of pulmonary function for patients with osteoporotic vertebral compression fractures after kyphoplasty. J Spinal Disord Tech 2007;20:221-5.

2. Kjensli A, Falch JA, Ryg M, et al. High prevalence of vertebral deformities in COPD patients: relationship to disease severity. Eur Respir J 2009;33:1018-24.

3. Graat-Verboom L, van den Borne BE, Smeenk FW, Spruit MA, Wouters EF. Osteoporosis in COPD outpatients based on bone mineral density and vertebral fractures. J Bone Miner Res 2011;26:561-8.

4. Krege JH, Kendler D, Krohn K, et al. Relationship between vertebral fracture burden, height loss, and pulmonary function in postmenopausal women with osteoporosis. J Clin Densitom 2015;18:506-11.

5. Watanabe R, Tanaka T, Aita K, et al. Osteoporosis is highly prevalent in Japanese males with chronic obstructive pulmonary disease and is associated with deteriorated pulmonary function. J Bone Miner Metab 2015;33:392-400.

6. Carter JD, Patel S, Sultan FL, et al. The recognition and treatment of vertebral fractures in males with chronic obstructive pulmonary disease. Respir Med 2008;102:1165-72.
7. Papaioannou A, Parkinson W, Ferko N, et al. Prevalence of vertebral fractures among patients with chronic obstructive pulmonary disease in Canada. Osteoporos Int 2003;14:913-7.

8. Nuti R, Siviero P, Maggi S, et al. Vertebral fractures in patients with chronic obstructive pulmonary disease: the EOLO Study. Osteoporos Int 2009;20:989-98.

9. Watanabe R, Shiraki M, Saito M, Okazaki R, Inoue D. Restrictive pulmonary dysfunction is associated with vertebral fractures and bone loss in elderly postmenopausal women. Osteoporos Int 2018;29:625-33.

10. Lombardi I Jr, Oliveira LM, Mayer AF, Jardim JR, Natour J. Evaluation of pulmonary function and quality of life in women with osteoporosis. Osteoporos Int 2005;16:1247-53.

11. Leech JA, Dulberg C, Kellie S, Pattee L, Gay J. Relationship of lung function to severity of osteoporosis in women. Am Rev Respir Dis 1990;141:68-71.

12. Schlaich C, Minne HW, Bruckner T, et al. Reduced pulmonary function in patients with spinal osteoporotic fractures. Osteoporos Int 1998;8:261-7.

13. Tanigawa N, Kariya S, Komemushi A, Nakatani M, Yagi R, Sawada S. Added value of percutaneous vertebroplasty: effects on respiratory function. AJR Am J Roentgenol 2012;198:W51-4.

14. Cimen OB, Ulubas B, Sahin G, Calikoglu M, Bagis S, Erdogan C. Pulmonary function tests, respiratory muscle strength, and endurance of patients with osteoporosis. South Med J 2003;96:423-6.

15. Kanis JA, McCloskey EV, Johansson H, Oden A, Melton LJ 3rd, Khaltaev N. A reference standard for the description of osteoporosis. Bone 2008;42:46775.

16. Siris ES, Miller PD, Barrett-Connor E, et al. Identification and fracture outcomes of undiagnosed low bone mineral density in postmenopausal women: results from the National Osteoporosis Risk Assessment. JAMA 2001;286:2815-22. 